\title{
Interferon- $\alpha$ Promotes the Expression of Cancer Stem Cell Markers in Oral Squamous Cell Carcinoma
}

Hailong Ma1\#, Shufang Jin,2\#, Wenyi Yang1, Zhuowei Tian¹, Shuli Liu¹, Yang Wang11, Ge Zhou³, Mei Zhao ${ }^{3}$, Shalva Gvetadze ${ }^{4}$, Zhiyuan Zhang1, ${ }^{1,}$, Jingzhou $\mathrm{Hu}^{1,} \bowtie$

1. Department of Oral Maxillofacial-Head and Neck Oncology, Shanghai Ninth People's Hospital, Shanghai Jiao Tong University School of Medicine, Shanghai Key Laboratory of Stomatology, Shanghai 200011, China

2. Department of Oral and Maxillofacial Surgery, The Second Affiliated Hospital Zhejiang University School of Medicine, Hangzhou 310009, China

3. Department of Head and Neck Surgery, The University of Texas MD Anderson Cancer Center, Houston, Texas 77030, USA

4. Central Research Institute of Dentistry and Maxillofacial Surgery, Congenital Maxillofacial Defects and Deformations, Timura Frunze 16, Moscow 119034, Russia

\#Hailong Ma and Shufang Jin contributed equally to this study.

$\square$ Corresponding authors: Jingzhou Hu, Department of Oral Maxillofacial-Head and Neck Oncology, Shanghai Ninth People's Hospital, Shanghai Jiao Tong University School of Medicine, Shanghai Key Laboratory of Stomatology, No 639, Zhizaoju Rd, Shanghai 200011, China. Tel: +86 021232716995160 ; Fax: +86 021 63136856; E-mail: huyayi@shsmu.edu.cn and Zhiyuan Zhang, Department of Oral Maxillofacial-Head and Neck Oncology, Shanghai Ninth People's Hospital, Shanghai Jiao Tong University School of Medicine, Shanghai Key Laboratory of Stomatology, No 639, Zhizaoju Rd, Shanghai 200011, China. Tel: +86 021 23271699 5160; Fax: +86 021 63136856; E-mail: zhzhy0502@163.com

(c) Ivyspring International Publisher. This is an open access article distributed under the terms of the Creative Commons Attribution (CC BY-NC) license (https://creativecommons.org/licenses/by-nc/4.0/). See http://ivyspring.com/terms for full terms and conditions.

Received: 2017.02.04; Accepted: 2017.05.01; Published: 2017.07.22

\begin{abstract}
Objectives: IFNa can stimulate an antitumor immune response and has a direct inhibition on cancer cells. This study is to test whether IFN $\alpha$ can activate dormant cancer stem cell (CSC) in oral squamous cell carcinoma (OSCC) to facilitate their elimination by chemotherapy.
\end{abstract}

Materials and methods: Nude mouse transplantation tumor model was established and administrated with IFN $\alpha$ and saline. The influence on CD44 and ALDHIAl expression under IFN $\alpha$ treatment was detected by in vivo experiments. Flow cytometry, western blot, and immunofluorescence were used to detect the expression of CD44 and ALDHIAI after INFa treatment in OSCC cell lines. Tumorsphere formation assay was conducted under incubation with IFN $\alpha$ for 2 weeks. Chromatin immunoprecipitation (ChIP) assays was used to examine the IFNa-induced transcriptional regulation of CD44 and ALDHIAI expression. That IFNa-primed enhanced killing effect of chemotherapy was evaluated by MTT and western blot.

Results: IFN $\alpha$ transcriptionally activated the expression of CD44 and ALDHIAI expression both in vivo and in vitro. IFNa-primed enhanced the cytotoxic inhibition effect of CDDP, erlotinib and nimotuzumab on OSCC cells.

Conclusion: These results suggest that IFNa could be administrated to patients prior to chemotherapeutic drugs, which will facilitate the killing of cancer stem cells in OSCC.

Key words: Interferon alpha; CD44; ALDH1A1; Oral squamous cell carcinoma

\section{Introduction}

Type I interferons (IFNs) mainly comprise IFNa and IFN $\beta$ proteins. IFNa has a major role not only in antiviral immune responses but also in the natural and the therapy-induced immunological control of virus-unrelated malignancies [1]. IFNa displays direct cytotoxicity on tumor cells in cell culture and preclinical studies [2]. IFNa has been reported to affect tumor cell proliferation, tumor lymph/ angiogenesis, and tumor metastasis [3]. IFNa induced by plasma dendritic cells ( $\mathrm{pDC}$ ) leads to tumor regression of melanoma, basal cell carcinoma, and $\mathrm{T}$ cell lymphoma [4]. Type I IFNs intervene all the 
phases of cancer immunoediting including elimination, equilibrium and escape [5]. IFNa can activate proliferation of DC and NK cells, enhance the cytotoxicity and survival of NK cells and $\mathrm{CD} 4^{+} \mathrm{T}$ cell, and promote tumor antigen expression of tumor cells [6-9]. Trials of IFNa therapies in solid malignancies such as melanoma, renal cell carcinoma and Kaposi sarcoma have achieved varied success [10]. Given its pleiotropic actions, IFNa is expected to not only stimulate an antitumor immune response, but also directly impact on cancer cell proliferation and survival, which together can achieve maximum therapeutic effect on cancer cells.

CSC has emerged as a critical factor for cancer chemoresistance, relapse and metastasis which often lead to treatment failure. CSC can undergo self-renewal and differentiation to promote the initiation and maintenance of tumor. CD44 and ALDH1A1 were considered as classical surface markers of CSC in head and neck squamous cell carcinoma (HNSCC) [11]. CD44 is a type I transmembrane glycoprotein that mediates cell-cell and cell-matrix interaction through affinity for hyaluronic acid. It is the one of the first markers to identify HNSCC CSCs [12]. OSCC CSCs can be also isolated on the basis of functional activities of ALDH1A1, which contributes to chemotherapy resistance [13]. Recently, more and more therapies were investigated to target the CSC population. However, CSCs usually lie dormant to avoid killing by chemotherapeutic drugs [14]. So how to promote the exit of CSCs out of the dormant stage is particularly important. Pre-treated/primed with IFNa can awaken dormant haematopoietic stem cells in vivo [15]. Whether IFNa can also activate dormant CSCs in OSCC to facilitate CSC's elimination by chemotherapy remains unclear.

In our current study we demonstrated that IFNa promoted the expression of CSC markers CD44 and ALDH1A1 in vitro and in vivo models of OSCC. Moreover, we showed that primed-IFNa can enhance the tumor-killing effect of chemotherapy. Our results strongly suggest that IFNa could be given to patients prior to chemotherapy in order to activate maximal therapeutic effects on CSC.

\section{Materials and methods}

\section{Materials}

Human recombinant IFNa was purchased from PeproTech company (Rocky Hill, NJ). A mouse monoclonal antibody against CD44 (CST) and a rabbit monoclonal antibody against ALDH1A1 (Abcam) were applied in this study. GAPDH and FITC-labeled secondary antibody were purchased from Proteintech (Rosemont, IL).

\section{In vivo experiments}

The nude mouse transplantation tumors were established with Cal27, a OSCC cell line, at $1 \times 10^{6}$ cells/point. IFNa was administrated $20000 \mathrm{IU} /$ day by subcutaneous injection after tumor formation, while the controls with normal saline (5 mice/group). Three weeks after administration, all the mice were sacrificed and the tumor tissues were preserved in liquid nitrogen and embedded in paraffin. Protein was extracted from the fresh tissue. Paraffin-cut sections were prepared for immunohistochemistry. The animal experiment was approved by Ethics Committee of Shanghai Ninth People's Hospital, Shanghai Jiao Tong University School of Medicine.

\section{RNA isolation and qPCR analysis}

Total RNA was extracted by TRIzol reagent (Invitrogen, Life Technologies) according to the manufacturer's instructions, and cDNA was generated using a Reverse Transcriptase Kit. Real-time quantitative PCR was performed in triplicate with Power PCR SYBR Green Master Mix (Takara Biotechnology, Dalian, China) using the ABI PRIAM Step-One Real-time PCR System (Applied Biosystems, Carlsbad, CA, USA) with results normalized to GAPDH expression. The relative expression was calculated using the $2^{-\triangle \Delta C T}$ method. CD44 primers: Forward: 5'- GGACCTTCATCCCAGT GACC -3', Reverse: 5'- GCTCCACCTTCTTGACTCC C - 3'. ALDH1A1 primers: Forward: 5' - ATCAAAGA AGCTGCCGGGAA -3', Reverse: 5' - GCATTGTCCAA GTCGGCATC -3'. GAPDH primers: Forward: 5'CCTCTGACTTCAACAGCGAC -3', Reverse: 5'TCCTCTTGTGCTCTTGCTGG -3 '

\section{Immunohistochemistry and immunofluorescence}

Xenograft tumors of nude mice were formalin-fixed and paraffin-embedded. Sections were heated by water bath at $100{ }^{\circ} \mathrm{C}$ with citrate buffer solution ( $\mathrm{pH}$ 6.0) for $20 \mathrm{~min}$ to retrieve antigen. CD44 at 1:50 dilution and ALDH1A1 at 1:400 dilution were incubated overnight at $4{ }^{\circ} \mathrm{C}$. Goat secondary antibody against mouse/rabbit immunoglobulin was incubated for 1 hour at room temperature. 3, 3-diaminobenzidine (DAB) detection was developed under light microscope. Every step of the wash was used phosphate buffered saline solution (PBS) for $5 \mathrm{~min}$ three times. The immunoreaction staining score was calculated by multiplying the percentage of positive cells and the staining intensity. For immunofluorescence, FITC-labeled secondary antibodies against mouse and rabbit were incubated for 1 hour in the cassette. The percentage of positive cells was counted under fluorescence microscope. Image-pro plus 6.0 
software was used to measure mean optical density for immunohistochemistry analysis.

\section{Cell cultures}

The OSCC cell lines HN4 and HN30 were kindly presented by the by the University of Maryland Dental School (USA). These cell lines were cultured in Dulbecco's modified Eagle's medium (DMEM) (Gibco, USA) supplemented with $10 \%$ fetal bovine serum, $1 \%$ glutamine, and $1 \%$ penicillin-streptomycin. All cells were cultured in a humidified atmosphere of $5 \% \mathrm{CO} 2$ at $37^{\circ} \mathrm{C}$.

\section{Flow cytometry analysis of CSC markers}

Flow cytometry was performed as described previously [16]. Briefly, HN4 and HN30 were treated with $0,2,20 \mathrm{ng} / \mathrm{ml}$ IFNa in 12 well-plate for $72 \mathrm{~h}$. The cells were collected, washed and incubated with CD44 and ALDH1 antibodies at 1:100 dilution. After 1 hour incubation, cells were washed and incubated with FITC-labeled secondary antibody for $30 \mathrm{~min}$. Then cells were washed twice and resuspended in $100 \mu \mathrm{l}$ of FACS buffer. The stained cells were analyzed on BD FORTASA flow cytometer. The final results were operated using FlowJo software (Tree Star).

\section{Western blot}

Western blot assay was performed as briefly described [17]. Cells were lysed and quantified. Protein samples ( $30 \mu \mathrm{g} /$ lane) were separated by $10 \%$ sodium dodecyl sulfate polyacrylamide gel electrophoresis (SDS-PAGE), and then electrophoretically transferred onto PVDF membranes using a wet transfer system (Bio-Rad, USA). The membranes were incubated overnight with the primary antibody of rabbit anti-ALDH1A1 monoclonal antibody (1:1 000) and mouse anti-CD44 monoclonal antibody (1:1 000). Antibodies against PARP, p-STAT1 (Tyr701), p-Akt (Ser473), p53, p-ERK1/2 (Thr202/Tyr204), and p-P38 (Thr180/Tyr182) were purchased from CST company and incubated at 1:1 000 dilution overnight. After incubating with fluorescent-based anti-rabbit/mouse IgG second antibody (Fermentas, Vilnius, Lithuania) at 1:10 000 dilution for $1 \mathrm{~h}$ in a dark place, immunoreactive bands were scanned and analyzed using Odyssey Infrared Imaging System (LICOR Biosciences, Lincoln, NE). GAPDH, $\beta$-actin, a-tubulin was used as internal control.

\section{Tumorsphere formation}

The sphere formation analysis was performed as described elsewhere [18]. Briefly, HN4 and HN30 cells were cultivated in the serum free RPMI-1640 medium containing $20 \mathrm{ng} / \mathrm{ml}$ of fibroblast growth factor, 20 $\mathrm{ng} / \mathrm{ml}$ epithelial growth factor, and $1 \% \mathrm{~B} 27$, with 0,2 , $20 \mathrm{ng} / \mathrm{ml}$ of IFNa in low adhesion plate. After two weeks, all the spheres were filtered through a 70 $\mu \mathrm{m}$-filter membrane. Only the spheres greater than 70 $\mu \mathrm{m}$ were counted. The sphere formation efficiency was calculated.

\section{Chromatin Immunoprecipitation (ChIP)}

ChIP was strictly operated according the protocol of SimpleChIP Enzymatic Chromatin IP kit (purchased from CST). After incubation with 20 $\mathrm{ng} / \mathrm{ml} \mathrm{IFNa}$ for $48 \mathrm{~h}, \mathrm{HN} 4$ was fixed with $1 \%$ formaldehyde for $10 \mathrm{~min}$ and quenched with glycine for $5 \mathrm{~min}$. Cells were washed twice in PBS and re-suspended in lysis buffer. Then the lysis was digested by micrococcal nuclease at $37^{\circ} \mathrm{C}$ for $20 \mathrm{~min}$ to length of approximately $150-900 \mathrm{bp}$. Digestion was terminated by the addition of $0.5 \mathrm{M}$ EDTA. Chromatin was sonicated at $30 \%$ output for $6 \times 10 \mathrm{sec}$. Clarify lysates by centrifugation at $10000 \mathrm{rpm}$ for $10 \mathrm{~min}$ at 4 ${ }^{\circ} \mathrm{C}$. The supernatant was diluted with ChIP buffer (1:9). Add the immunoprecipitating antibodies into $500 \mu \mathrm{l}$ of the diluted chromatin. For the positive control Histone $\mathrm{H} 3$ rabbit $\mathrm{mAb}$, add $10 \mu \mathrm{l}$ to the IP sample. For the negative control normal rabbit IgG, add $5 \mu$ to the IP sample. $5 \mu \mathrm{l}$ p-STAT1 (Try 701) (CST) was added into the sample. Incubate IP samples overnight at $4{ }^{\circ} \mathrm{C}$ with rotation. Add $30 \mu$ of ChIP Grade Protein G Magnetic beads and incubate for $2 \mathrm{~h}$ at $4{ }^{\circ} \mathrm{C}$ with rotation. Wash the immunoprecipitated chromatin with three times low salt wash and once high salt wash. Eluted chromatin was reversed cross-links by adding $6 \mu \mathrm{l} 5 \mathrm{M} \mathrm{NaCl}$ and $2 \mu \mathrm{l}$ proteinase $\mathrm{K}$ into every samples overnight at $65^{\circ} \mathrm{C}$. Reverse cross-linked DNA was purified by spin columns. Quantitative polymerase chain reaction analysis of purified ChIP DNA (ChIP-qPCR) was performed to calculate the enrichment percentage of promoter region using $2^{-\Delta \Delta}{ }^{-}$T formula. The CD44 promoter region primers: Forward: 5' - ATCTAGGTG TTCTAGCTCCTGAATC - 3 ', Reverse: 5' - CTACCATT CCTAGAGAAGGGAGTC - $3^{\prime}$. The ALDH1A1 promoter region primers: Forward: 5'- CTTGGTCCCTAG GTTCTCACAA -3', Reverse: 5' - CACTTAAGCAGAT CTTTTCTGCC $-3^{\prime}$.

\section{Cell viability assay}

$\mathrm{HN} 4$ and HN30 was incubated with $0,2 \mathrm{ng} / \mathrm{ml}$ IFNa for $48 \mathrm{~h}$. After INFa primed, 3000 cells per well were seeded in the 96-well plates. They were then treated with the indicated concentration of drugs in $10 \%$ FBS DMEM containing CDDP, erlotinib, nimotuzumab for $72 \mathrm{~h}$. The total cell numbers were assessed after incubation with $20 \mu \mathrm{l}$ of MTT for $4 \mathrm{~h}$. The optical density (OD) of each well was measured using a microplate reader at $490 \mathrm{~nm}$, and the OD values are presented as the means \pm SD. 


\section{Statistical analysis}

Statistical analysis was performed using the software of GraphPad Prism version 6 for Windows (GraphPad Software, San Diego, CA, USA). All values
A

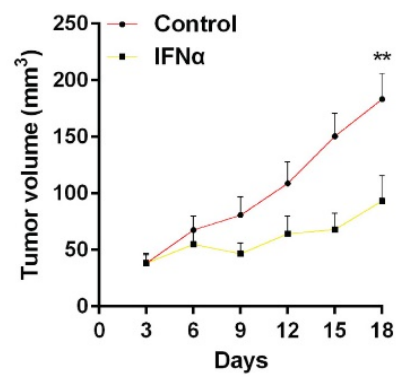

C

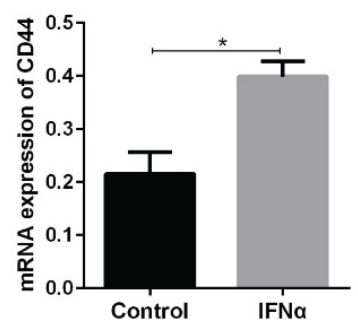

D

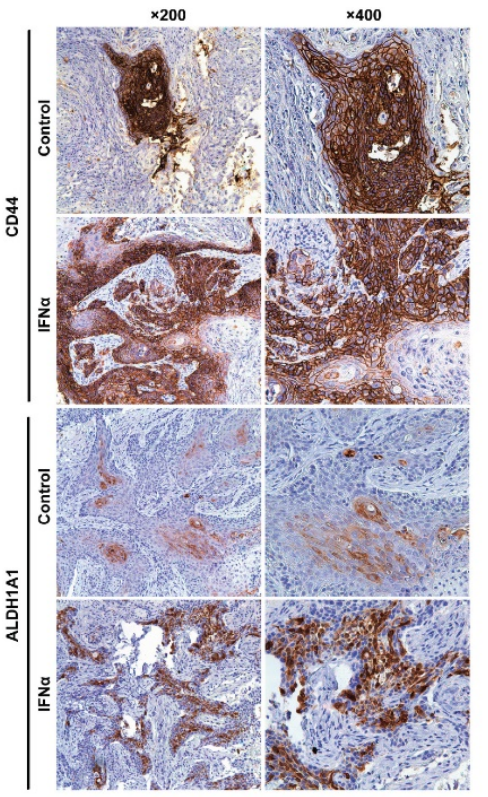

B
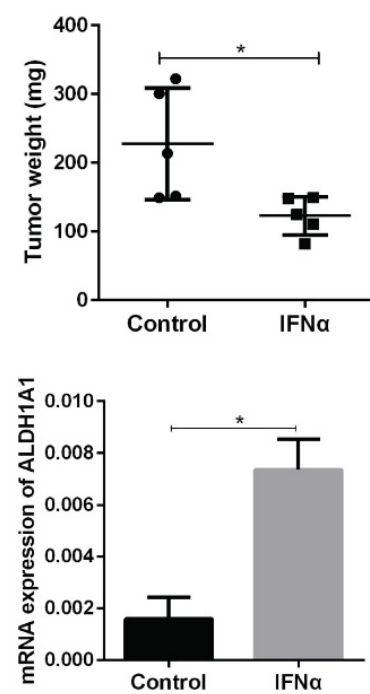

are expressed as mean $\pm S D$, and Student's $t$ test was performed to assess the statistical significance of difference. $\mathrm{P}<0.05$ was considered statistically significant.

\section{Results}

\section{IFN $\alpha$ induces the expression of CSC markers CD44 and ALDH1A1 in vivo}

From in vivo experiment, we have confirmed the tumor inhibitory effect of IFNa in OSCC xenograft in nude mice. The tumor volume in IFNa group was much smaller than control $(104.9 \pm 22.1$ vs $60.84 \pm 7.88, \mathrm{P}<0.01$. Figure 1A). The tumor weight in treatment group also decreased compared to control $(227.5 \pm 36.4$ vs $122.9 \pm 12.5, \mathrm{P}=0.02$. Figure 1B). PCR assay demonstrated that mRNA expression of CD44 and ALDH1A1 was also significantly elevated in treatment group (Figure 1C). Immunohistochemistry (IHC) assay indicated that CD44 localizes on cell membrane, while ALDH1A1 is in the cytoplasm (Figure 1D). The relative mean optical density (MOD) of CD44 that represents the IHC score for CD44 expression was significantly higher in IFNa-treatment tumors than that in the control $(5.667 \pm 0.409$ vs 1.100 $\pm 0.208, P<0.01)$. Similarly, MOD of ALDH1A1 was also much higher in IFNa treatment group. Our western blot showed that CD44 and ALDH1A1 proteins was elevated in tumors of random three mice of the treatment group compared to the untreated control (Figure 1E).

\section{IFN $\alpha$ upregulates the expression of CD44 and ALDHIAl and promotes tumorsphere formation in vitro}

To investigate the impact of INF $\alpha$ on expression of markers in HNSCC cells, we treated HN4 and HN30 cells with IFNa and examined the expression CD44 and ALDH1A1 using flow cytometry. As shown in Figures 2A-B, after $72 \mathrm{~h}$ of treatment the mean fluorescence intensity (MFI) of CD44 and ALDH1A1 were increased in an INF $\alpha-$ dependent manner. Similarly, the percentage of CD44 and ALDH1A1 positive cells were significantly elevated after treatment (Figures 2C-D), suggesting that IFN $\alpha$ upregulated CD44 and ALDH1A1 expression in both HN4 and HN30 cells. samples from OSCC mouse xenograft with and without IFN $\alpha$ treatment. (D) IHC staining
Figure 1. IFN $\alpha$ induces the expression of CSC markers CD44 and ALDH1A1 in vivo. (A) The tumor volume was measured every three days with vernier caliper and calculated as $a \times b^{2} / 2$ (a: length, b: width). (B) The tumor weight in each group was measured after administration. (C) Real-time PCR analyses of CD44 and ALDHIAI mRNA in tumor of CSC markers in OSCC xenograft tumor samples with and without IFN $\alpha$ treatment. Relative MOD of CD44 and ALDHIA1 in IHC staining was analyzed. (E) Western blot of tumor samples from OSCC mouse xenograft with and without IFN $\alpha$ treatment. Magnification: $\times 200, \times 400$. 


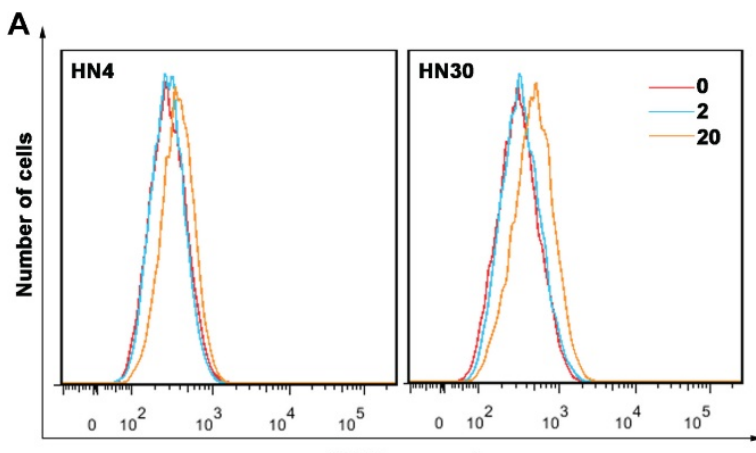

B
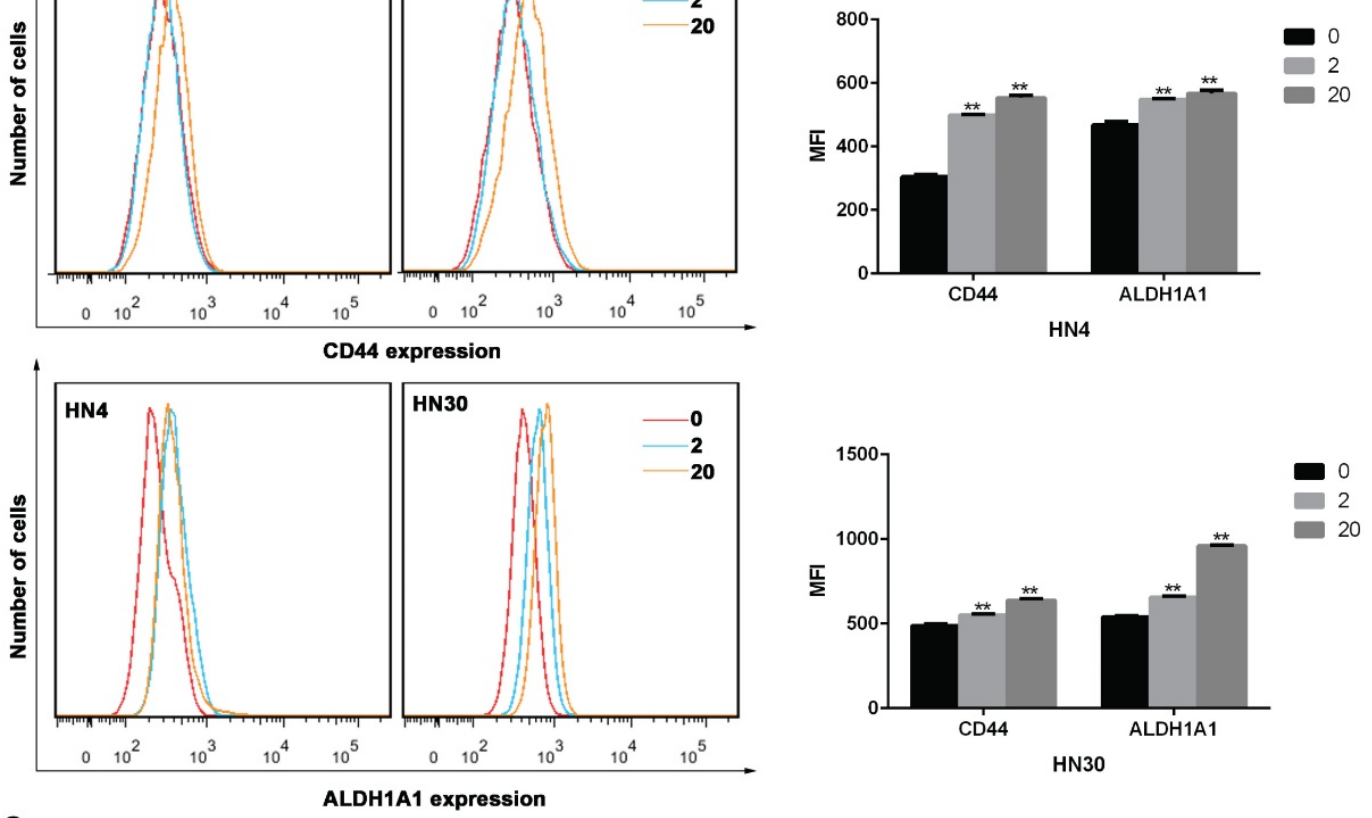

C

CD44

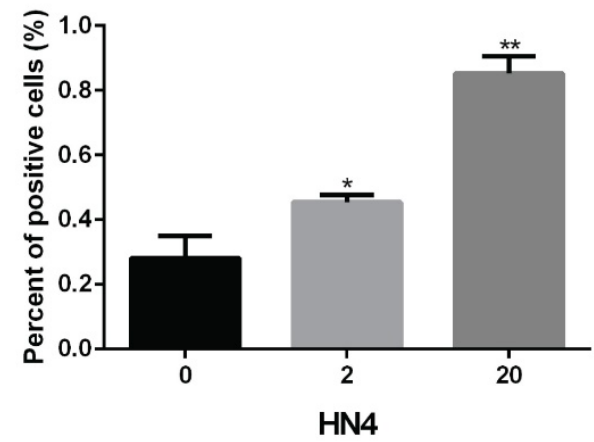

D

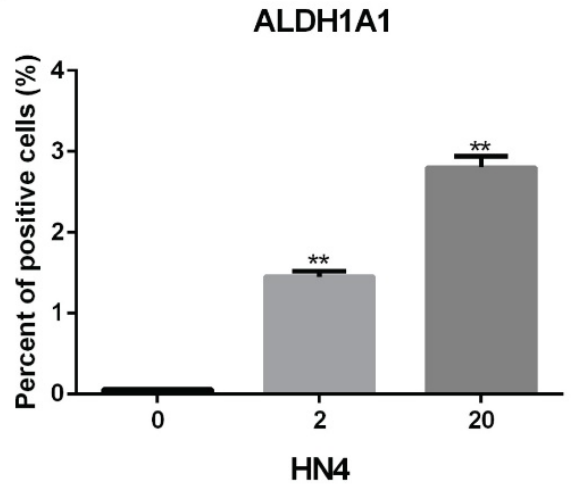

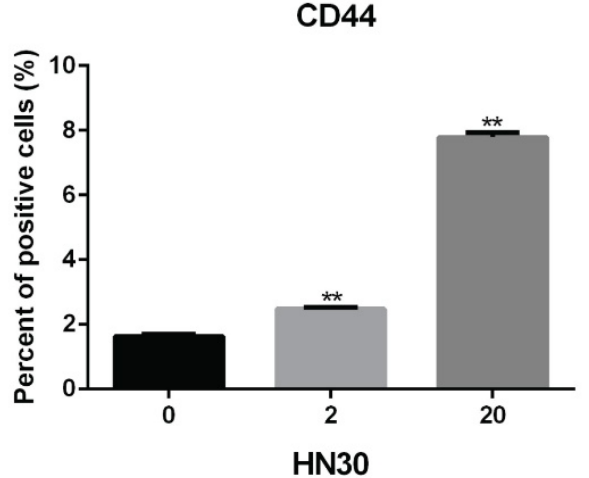

ALDH1A1

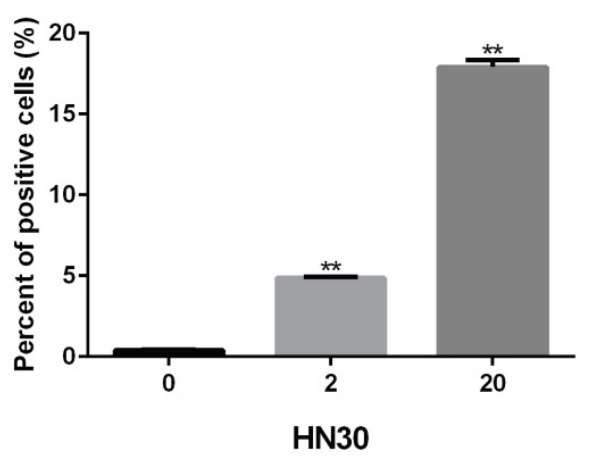

Figure 2. IFN $\alpha$ upregulates the expression of CD44 and ALDHIAl in vitro. (A) Flow cytometry analyses of CD44 and ALDHIAl expression in HN4 and HN30 cells in the absence (0) and presence of INF $\alpha$ treatment $(2 \mathrm{ng} / \mathrm{ml} ; 20 \mathrm{ng} / \mathrm{ml})$ for $48 \mathrm{~h}$. (B) Mean fluorescence intensity (MFI) of CD44 and ALDHIAl in the absence (0) and presence of INF $\alpha$ treatment ( $2 \mathrm{ng} / \mathrm{ml} ; 20 \mathrm{ng} / \mathrm{ml})$ for $48 \mathrm{~h}$. (C-D) Percent of positive CD44 and (C) ALDHIAI (D) cells in HN4 and HN30 before and after INF $\alpha$ treatment.

Consistent with the results from flow cytometry, western blot also indicated that the CD44 and ALDH1A1 expression were increased after IFNa incubation (Figure 3A), while immunofluorescence assay showed that CD44 and ALDH1A1 positive cells were enriched after $20 \mathrm{ng} / \mathrm{ml}$ IFNa treatment (Figure 3B). CD44 located on the cell membrane (Figure 3C), whereas ALDH1A1 located in the cytoplasm of HN4 
and HN30 cells (Supplementary Figure 1). Together, our results demonstrated that IFNa upregulated CD44 and ALDH1A1 expression in both HN4 and HN30 cells in a dose-dependent manner.

Sphere-forming capacity of tumor cells correlates directly with CSC phenotype and thus tumorigenic efficiency of the cancer cells can be determined based on the number of spheres that originate from specific number of seeded cells [19]. HN4 and HN30 were examined for their ability to form tumorspheres in vitro (Figure $\mathbf{4 A}$ ). The number of tumorsphere

A
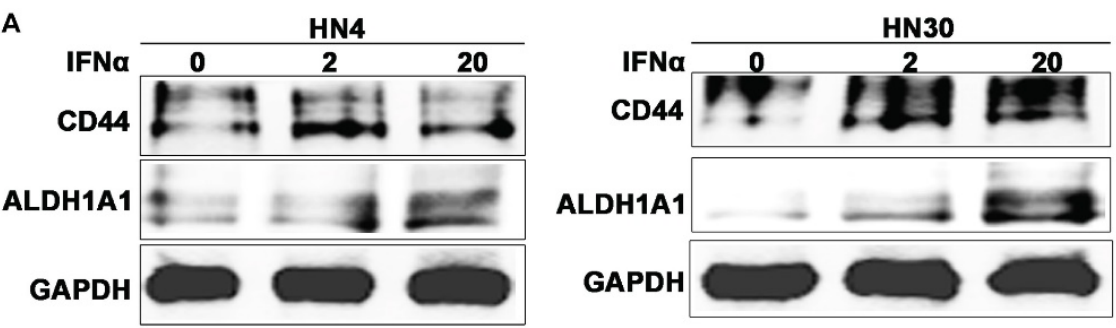

B

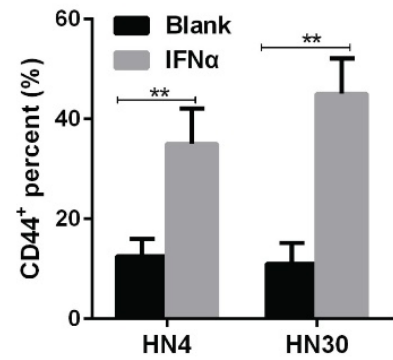

C

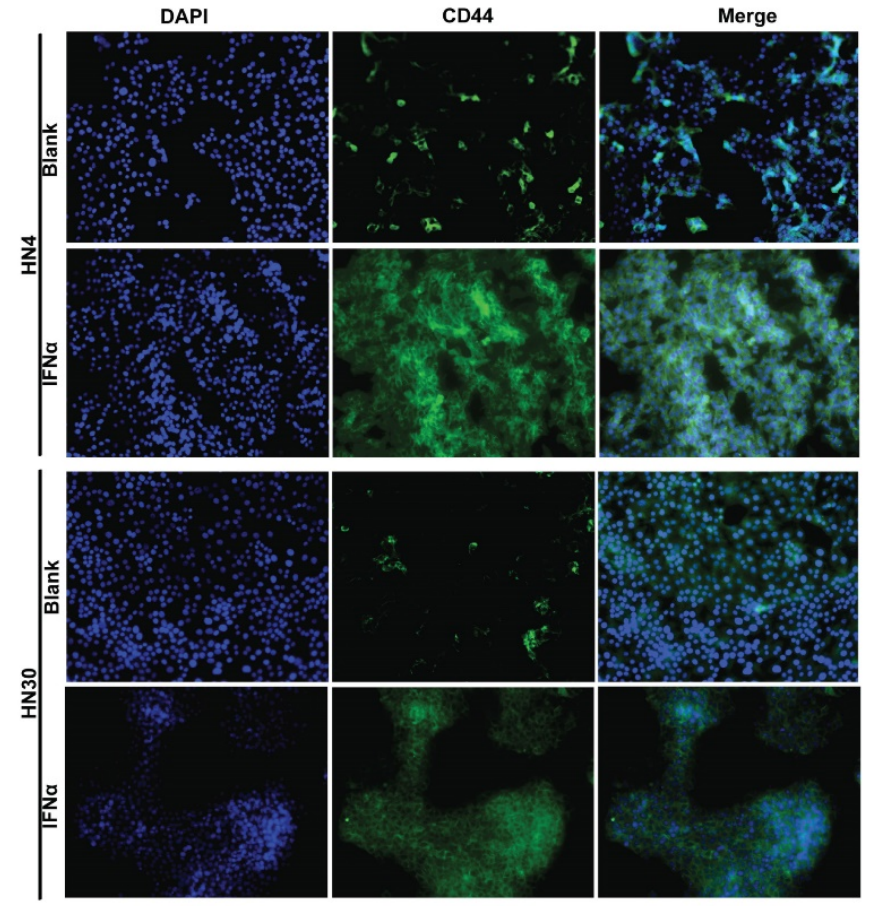

Figure 3. IFN $\alpha$ upregulates the expression of CD44 and ALDHIAl in OSCC cells. (A) Western blot of CD44 and ALDHIAl in HN4 and HN30 cells before and after IFN $\alpha$ treatment $(2 \mathrm{ng} / \mathrm{ml} ; 20 \mathrm{ng} / \mathrm{ml})$ for $48 \mathrm{~h}$. (B) Immunofluorescent (IF) assay of CSC markers in $\mathrm{HN} 4$ and $\mathrm{HN} 30$ cells before and after IFN $\alpha$ treatment. (C) Representative images of IF staining of CD44 in HN4 and HN30 in the presence and absence of IFNa treatment. Magnification: $\times 200$. increased after IFNa stimulation for two weeks (Figure 4B). Furthermore, sphere forming efficiency also increased in $\mathrm{HN} 4$ and HN30 with the treatment of IFNa (Figure 4C), suggesting that IFNa promoted tumorsphere formation of HN4 and HN30 cells.

\section{IFN $\alpha$ transcriptionally activates CD44 and ALDHIAI expression through inducing the binding of phosphorylated (activated) signal transducer and activator of transcription 1 (STAT1) to CD44 and ALDHIAI promoters}

In response to IFNa stimulation, STAT1 forms heterodimers with STAT2 that bind the Interferon-Stimulated Response Element (IRSE) promoter element [20]. So we speculated that IFNa transcriptionally activated CD44 and ALDH1A1 expression through similar mechanism. In support of this, ChIP-PCR assay indicated that Tyr-701 phosphorylated STA$\mathrm{T} 1$ bound to the promoter region of CD44 and ALDH1A1 under IFNa stimulation in HN4 cells (Figure 5A), whereas no obvious binding was detected in normal IgG control (Figure 5B). This result strongly suggests that IFNa can induce phosphorylation (activation) of STAT1, leading its binding to the upstream promoters of $C D 44$ and ALDH1A1 and transcriptionally activate the expression of CD44 and ALDH1A1 in HN4 cells.

\section{Primed-IFN $\alpha$ can enhance the tumor-killing effect of CDDP, Erlotinib and Nimotuzumab}

From the above results, we confirmed the activation of CSC markers by IFNa in OSCC cells. We hypothesized that the tumor cells would be more sensitive to chemotherapy after activation of CSC. To test this, HN4 and HN30 were treated with $20 \mathrm{ng} / \mathrm{ml}$ IFNa for 48 hour, and then seeded in 96-well plates and further treated with CDDP (a classic cytotoxic drug for OSCC), Erlotinib and Nimotuzumab (epidermal growth factor receptor inhibitors), respectively. Nimotuzumab binds to the epidermal growth factor receptor (EGFR) and Erlotinib is a tyrosine 
kinase inhibitor of EGFR in HNSCC [21]. Our result showed that IFNa sensitized the cells to the tumor inhibitory effect of CDDP (Figure 6A), Erlotinib (Figure 6C), and Nimotuzumab (Figure 6E), respectively. Combination of primed-INFa treatment with 1 $\mu \mathrm{g} / \mathrm{ml}$ CDDP, PARP, p-ERK and p-P38 were elevated while p-Akt and p53 decreased (Figure 6B). Combination of primed-INF $\alpha$ treatment with $5 \mu \mathrm{M}$ Erlotinib, p53 decreased while p-STAT1, p-ERK and p-P38 increased (Figure 6D). In addition, PARP, p-Akt, p-P38 and p-ERK were increased and only p53 decreased under $200 \mu \mathrm{g} / \mathrm{ml}$ Nimotuzumab (Figure 6F). Taken together, our results demonstrated that OSCC cells primed with IFNa can be efficiently eliminated by treatment of CDDP, Erlotinib and Nimotuzumab in vitro.

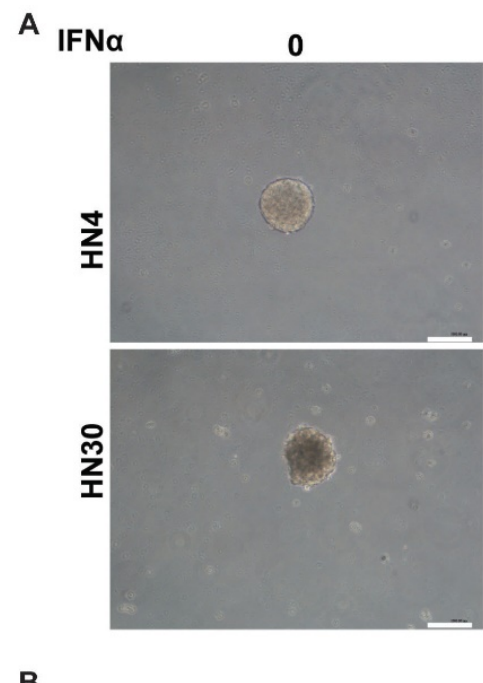

B

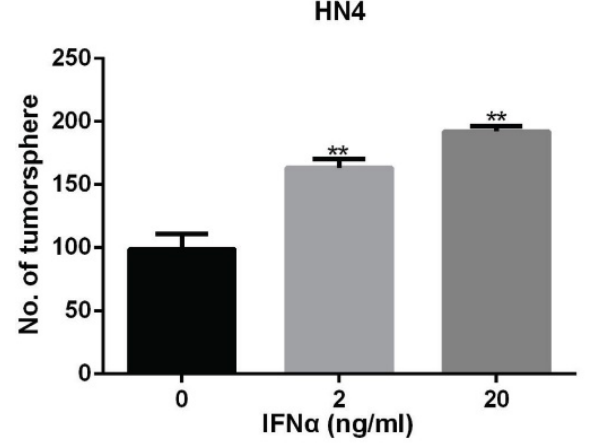

C

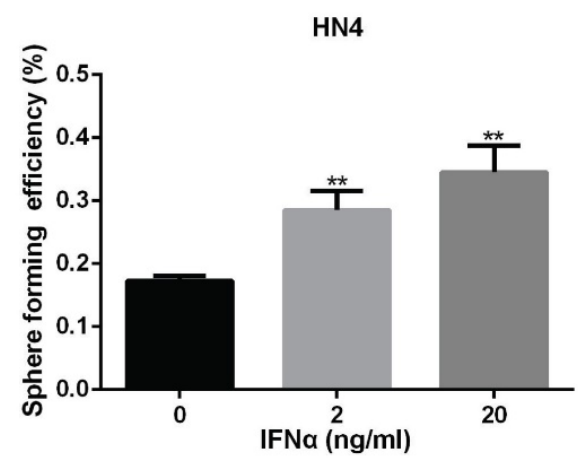

2
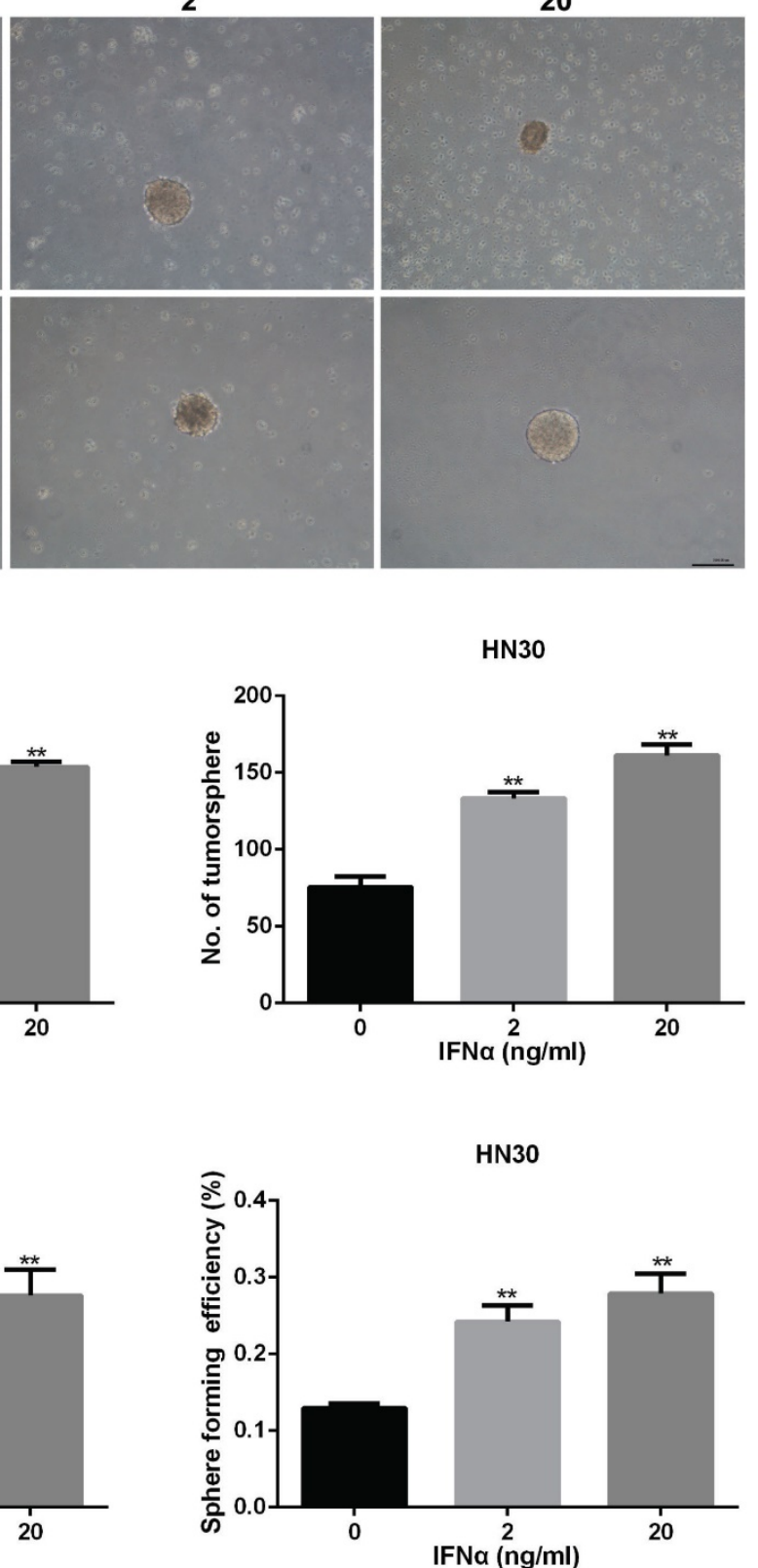

Figure 4. IFN $\alpha$ enhances tumorsphere formation ability in HN4 and HN30 cells. (A) Representative images of tumorsphere formation in conditioned medium with indicated concentration of IFN $\alpha(2 \mathrm{ng} / \mathrm{ml} ; 20 \mathrm{ng} / \mathrm{ml})$ for 2 weeks. Scar bar: $1 \mathrm{~mm}$. (B) Quantitation of the number of tumorsphere formation in HN4 and HN30 cells in the presence and absence of IFN $\alpha$ incubation. (C) Sphere forming efficiency of HN4 and HN30 cells in the presence and absence of IFN $\alpha$ incubation. 
A

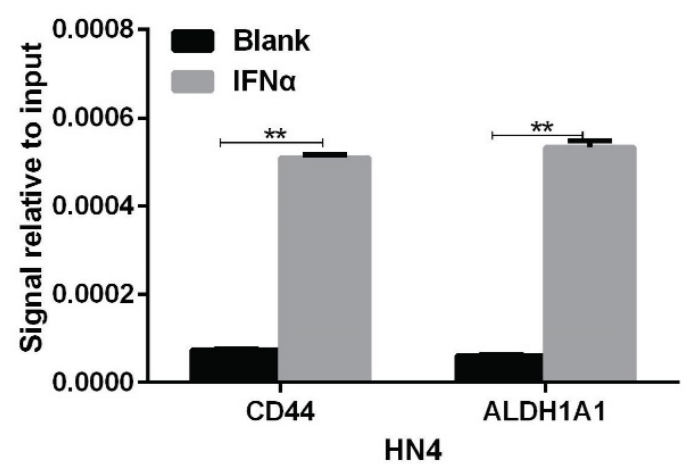

B

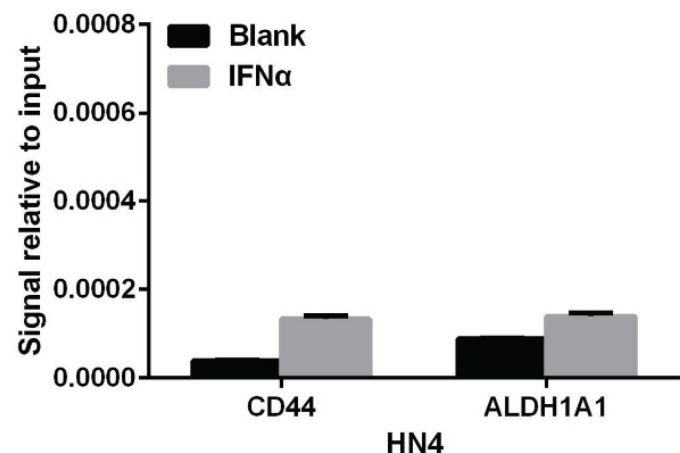

Figure 5. IFN $\alpha$ transcriptionally activates CD44 and ALDHIAI expression through inducing the binding of phosphorylated STATI to CD44 and ALDHIAI promoters. (A) ChIP assay of p-STATI (pTry701) in promoter regions of CD44 and ALDHIAl were under IFN $\alpha$ treatment for $48 \mathrm{~h}$. (B) ChIP assay of control $\lg G$ in promoter regions of CD44 and ALDHIAI were under IFN $\alpha$ treatment.

\section{Discussion}

In this study, we demonstrated that IFNa can upregulated CSC markers of oral cancer cells and primed-IFNa sensitized the therapeutic effect of CDDP, Erlotinib and Nimotuzumab. CSCs have stem features such as self-renewal, high migration capacity, drug resistance, high ability of tumorigenicity. In OSCC, CSCs have been increasingly shown to have an integral role in tumor initiation, disease progression, metastasis and treatment resistance [22]. So targeting for the eradication of CSCs was critical. In haematopoietic stem cells, three factors-granulocyte colony-stimulating factor, IFNa and arsenic trioxide have been shown to efficiently activate dormant stem cells and thereby could break their resistance to anti-proliferative chemotherapy [14]. IFNa upregulated the expression of the CSC markers CD24, CD44 and CD133 in in vitro and in vivo models of pancreatic ductal adenocarcinoma [23], which was consistent with our results. Our results suggested that IFNa promoted the expression of CSC markers through transcription activation in HN4 cells (Figure 5). However, we failed to detect IFNa-induced binding of STAT1 to the promoter of CSC markers in HN30 cells (data not shown). The difference may come from cellular heterogeneity, such as wild-type p53 expression in HN30 [24]. IFNa may promote the expression of ALDH1A1A in HN30 through other STAT members, which perhaps can bind the promoter region of ALDH1A1. Furthermore, IFNa may promote it through post-transcriptional regulation, posttranslational regulation and the modulation by interacting proteins. A number of studies have demonstrated that IFNa had a direct cytotoxic effect on various tumors and acted as a chemo-sensitizing agent $[25,26]$. So IFNa can enrich CSCs in tumors while directly kill the bulk of the tumor cells. This distinctive feature of IFNa can provide a new method for CSC-targeted therapy in OSCC.

While CD44 and ALDH1A1 were used as biomarkers for CSC in OSCC in our study, BMI-1 is another stem cell-related biomarker involved in the carcinogenesis in HNSCC [12]. CD133 antigen, known as prominin-1 has been also identified as a CSC marker in several cancers and particularly in the laryngeal cancer [27]. However, the combined use of ALDH1 and CD44 is more relevant for identifying CSC-like populations as it is more selective than any other marker used alone [28]. ALDH expression was seen in only $1 \%-7.8 \%$ of HNSCC cells, and were able to form tumors with injections of as few as 500 cells into mice. While, HNSCC tumors shown a proportion of CD44+ cells ranging from $0.1 \%$ to $41.72 \%$ of the tumor population [27]. Combination of CD44 and ALDH1A1 can identify almost all the CSC population in HNSCC, which had strong capacity of tumorigensis. So two generally accepted biomarkers CD44 and ALDH1A1, were adopted in our investigation.

Accumulating evidences have demonstrated that IFNa possessed chemo-sensitizing characteristics under different drugs. Radical surgery followed by IFNa and 5-FU combination chemotherapy offers possibility of long-term survival despite massive hepatocellular carcinoma with multiple intrahepatic metastases [29, 30]. IFNa pre-treatment followed by a combination of IFNa plus Erlotinib significantly enhanced the sensitivity of colon cancer cell lines [31]. IFNa enhanced the sensitivity to an anti-epidermal growth factor receptor monoclonal antibody (Nimotuzumab) in a human lung cancer cell line with intermediate expression of the receptor [32]. Low dose of IFNa intensified antineoplastic effect of a histone deacetylase -inhibitor, valproic acid, and the mammalian target of rapamycin inhibitor everolimus in prostate cancer cells [33]. It should be noted that in these studies IFNa was administrated together with chemoradiotherapy. Patients can benefit from 
unspecific activation of the immune system through IFNa to obtain better outcomes. While the future of enrichment of CSCs was implied with IFNa-primed in our study, only CDDP, Erlotinib and Nimotuzumab
A

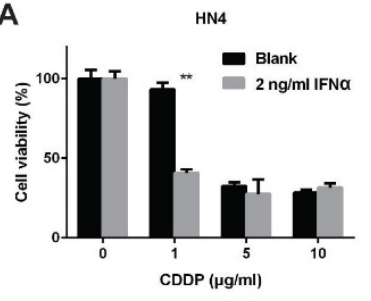

HN30

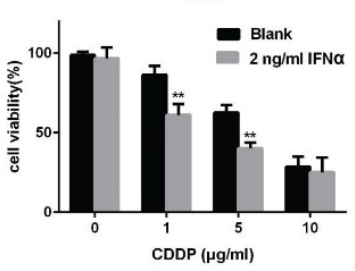

C

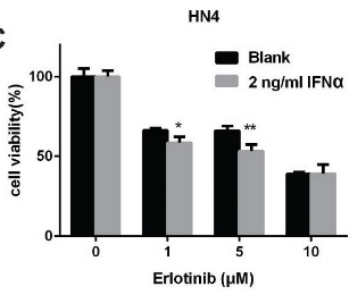

HN30

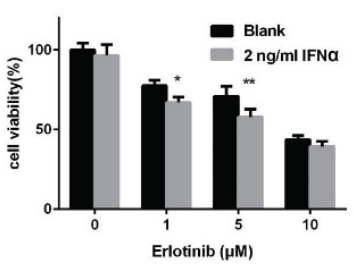

HN4

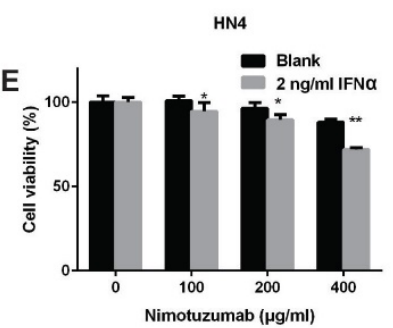

HN30

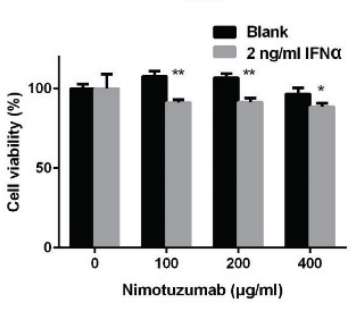

B

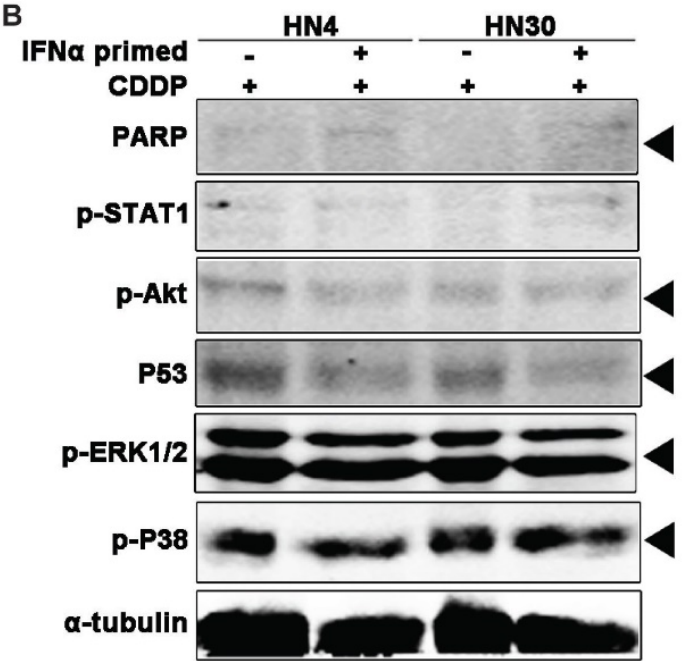

D

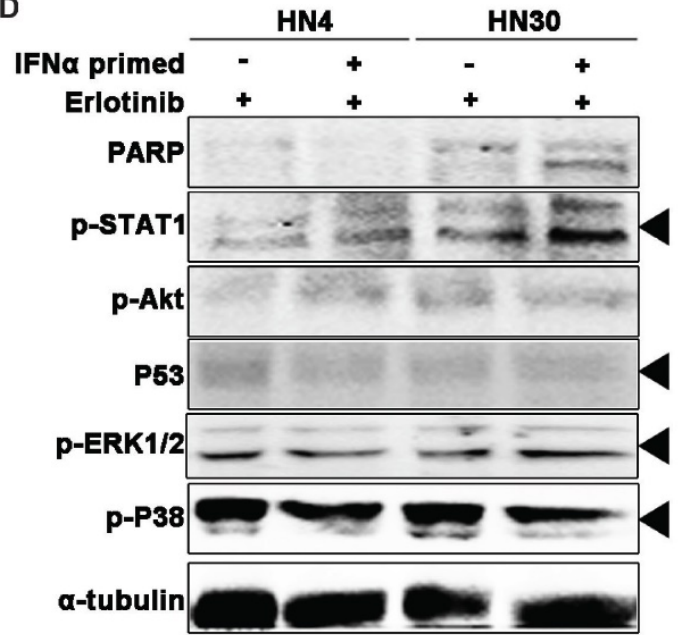

$\mathbf{F}$

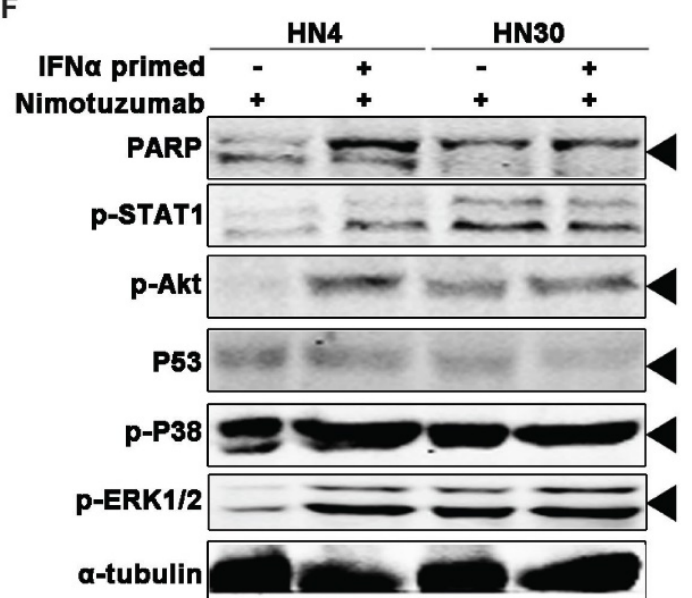

Figure 6. IFN $\alpha$ pretreatment enhances the sensitivity of OSCC cells to chemotherapy. (A) Cell viability of OSCC cells pretreated with $2 \mathrm{ng} / \mathrm{ml}$ IFN $\alpha$, followed by CDDP treatment at different concentration. (B)

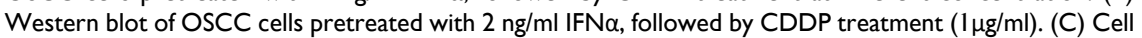

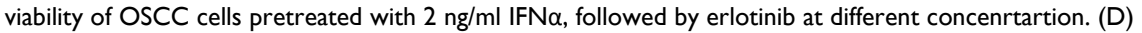

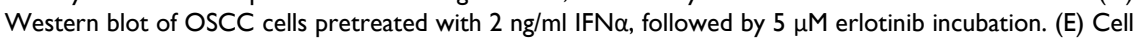

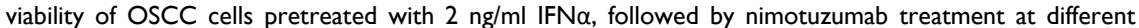

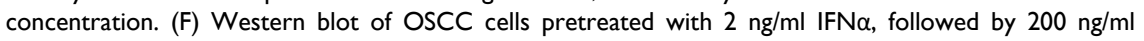
nimotuzumab incubation for $48 \mathrm{~h}$.

can be sensitized with pretreated with low dose IFNa in HN4 and HN30 (Figure 6). This synergistic effect should be further explored in in vitro study. Our results showed that p38 and ERK1/2 were activated after IFNa pretreatment, while p53 decreased in OSCC cells (Figure 6B, D, F). The activation of Akt depend the chemotherapies. p38 could be strongly activated by a wide variety of environmental and cellular stresses or by inflammatory cytokines [34]. Under certain conditions, ERK1/2 can also have pro-apoptotic functions which has lead this pathway to receive significant attention for development of cancer therapies [35]. Poly (ADP-ribose) polymerase (PARP) can be activated in cells experiencing stress and/or DNA damage. Our results showed that PARP was also elevated and activated under IFNa-primed and chemotherapy treatment. It should be noted that STAT1 that would be directly activated by IFNa was not significantly activated because of IFNa withdrawal under IFNa-primed and chemotherapy treatment. Taken together, IFNa could be given prior to chemotherapy to enrich the CSC and followed with concurrent with drugs to activate immune system. This hypothesis should be confirmed in future preclinical studies.

In summary, our studies indicate that IFNa can activate dormant CSCs in OSCC to facilitate the killing of CDDP, Erlotinib and Nimotuzumab. Our results provide experimental evidences and new treatment strategy for application of IFNa in OSCC.

\section{Supplementary Material}

Figure S1. http:/ / www.jcancer. org/v08p2384s1.pdf 


\section{Acknowledgements}

This study was supported by grants from Project of National Natural Science Foundation of China (Grant No. 31140007, 81472516), Natural Science Foundation of Shanghai (No. 14ZR1424200), Shanghai Leading Academic Discipline Project (No. S30206), and Doctoral Innovation Fund of Shanghai Jiao Tong University School of Medicine (BXJ201728).

\section{Competing Interests}

The authors declare no conflicts of interest.

\section{References}

[1]. Zitvogel L, Galluzzi L, Kepp O, et al. Type I interferons in anticancer immunity. Nat Rev Immunol. 2015;15:405-14

[2]. Iacopino F, Ferrandina G, Scambia G, et al. Interferons inhibit EGF-stimulated cell growth and reduce EGF binding in human breast cancer cells. Anticancer Res. 1996;16:1919-24

[3]. Demoulin S, Herfs M, Delvenne P, et al. Tumor microenvironment converts plasmacytoid dendritic cells into immunosuppressive/tolerogenic cells: insight into the molecular mechanisms. J Leukoc Biol. 2013;93:343-52.

[4]. Urosevic M, Dummer R, Conrad C, et al. Disease-independent skin recruitment and activation of plasmacytoid predendritic cells following imiquimod treatment. J Natl Cancer Inst. 2005;97:1143-53.

[5]. Schreiber RD, Old LJ, and Smyth MJ. Cancer immunoediting: integrating immunity's roles in cancer suppression and promotion Science. 2011:331:1565-70.

[6]. Greiner JW, Hand PH, Noguchi P, et al. Enhanced expression of surface tumor-associated antigens on human breast and colon tumor cells after recombinant human leukocyte alpha-interferon treatment. Cancer Res. 1984:44:3208-14.

[7]. Schiavoni G, Mattei F, and Gabriele L. Type I Interferons as Stimulators of DC-Mediated Cross-Priming: Impact on Anti-Tumor Response. Front Immunol. 2013;4:483.

[8]. Brinkmann V, Geiger T, Alkan S, et al. Interferon alpha increases the frequency of interferon gamma-producing human CD4+ T cells. J Exp Med. 1993;178:1655-63.

[9]. Biron CA, Nguyen KB, Pien GC, et al. Natural killer cells in antiviral defense: function and regulation by innate cytokines. Annu Rev Immunol. 1999;17:189-220.

[10]. Parker BS, Rautela J, and Hertzog PJ. Antitumour actions of interferons: implications for cancer therapy. Nat Rev Cancer. 2016;16:131-44.

[11]. Dionne LK, Driver ER, and Wang XJ. Head and Neck Cancer Stem Cells: From Identification to Tumor Immune Network. J Dent Res. 2015;94:1524-31.

[12]. Prince ME, Sivanandan R, Kaczorowski A, et al. Identification of a subpopulation of cells with cancer stem cell properties in head and neck squamous cell carcinoma. Proc Natl Acad Sci U S A. 2007;104:973-8.

[13]. Wei Y, Wu S, Xu W, et al. Depleted aldehyde dehydrogenase 1A1 (ALDH1A1) reverses cisplatin resistance of human lung adenocarcinoma cell A549/DDP. Thorac Cancer. 2016;

[14]. Trumpp A, Essers M, and Wilson A. Awakening dormant haematopoietic stem cells. Nat Rev Immunol. 2010;10:201-9.

[15]. Essers MA, Offner S, Blanco-Bose WE, et al. IFNalpha activates dormant haematopoietic stem cells in vivo. Nature. 2009:458:904-8.

[16]. Fang S, Huang Y, Wang S, et al. IL-17A Exacerbates Fibrosis by Promoting the Proinflammatory and Profibrotic Function of Orbital Fibroblasts in TAO. J Clin Endocrinol Metab. 2016;101:2955-65.

[17]. Ma HL, Jin SF, Tao WJ, et al. Overexpression of stathmin/oncoprotein 18 correlates with poorer prognosis and interacts with p53 in oral squamous cell carcinoma. J Craniomaxillofac Surg. 2016;44:1725-32.

[18]. Yan M, Yang X, Wang L, et al. Plasma membrane proteomics of tumor spheres identify CD166 as a novel marker for cancer stem-like cells in head and neck squamous cell carcinoma. Mol Cell Proteomics. 2013;12:3271-84.

[19]. Wu J, Mu O Thiviyanathan V, et al Cancer stem cells are enriched in Fanconi anemia head and neck squamous cell carcinomas. Int J Oncol. 2014;45:2365-72.

[20]. Katze MG, He Y, and Gale M, Jr. Viruses and interferon: a fight for supremacy. Nat Rev Immunol. 2002;2:675-87.

[21]. Zibelman $M$ and Mehra R. Overview of Current Treatment Options and Investigational Targeted Therapies for Locally Advanced Squamous Cell Carcinoma of the Head and Neck. Am J Clin Oncol. 2016;39:396-406.

[22]. Mery B, Guy JB, Espenel S, et al. Targeting head and neck tumoral stem cells: From biological aspects to therapeutic perspectives. World J Stem Cells. 2016;8:13-21.

[23]. Zhu Y, Karakhanova S, Huang X, et al. Influence of interferon-alpha on the expression of the cancer stem cell markers in pancreatic carcinoma cells. Exp Cell Res. 2014;324:146-56.
[24]. Song X, Xia R, Li J, et al. Common and complex Notch1 mutations in Chinese oral squamous cell carcinoma. Clin Cancer Res. 2014;20:701-10.

[25]. Vitale G, van Eijck CH, van Koetsveld Ing PM, et al. Type I interferons in the treatment of pancreatic cancer: mechanisms of action and role of related receptors. Ann Surg. 2007;246:259-68.

[26]. Kang Y and Perry RR. Effect of alpha-interferon on P-glycoprotein expression and function and on verapamil modulation of doxorubicin resistance. Cancer Res. 1994:54:2952-8.

[27]. Wei XD, Zhou L, Cheng L, et al. In vivo investigation of CD133 as a putative marker of cancer stem cells in Hep-2 cell line. Head Neck. 2009;31:94-101.

[28]. Krishnamurthy S, Dong Z, Vodopyanov D, et al. Endothelial cell-initiated signaling promotes the survival and self-renewal of cancer stem cells. Cancer Res. 2010;70:9969-78.

[29]. Tanaka K, Yabushita Y, Nakagawa K, et al. Debulking surgery followed by intraarterial 5-fluorouracil chemotherapy plus subcutaneous interferon alfa for massive hepatocellular carcinoma with multiple intrahepatic metastases: a pilot study. Eur J Surg Oncol. 2013;39:1364-70.

[30]. Yamashita T, Arai K, Sunagozaka H, et al. Randomized, phase II study comparing interferon combined 5-fluorouracil plus cisplatin hepatic arterial infusion with interferon combined 5-fluorouracil hepatic arterial infusion in patients with advanced hepatocellular carcinoma. J Clin Oncol. 2009;27:4588.

[31]. Yang JL, Qu XJ, Russell PJ, et al. Interferon-alpha promotes the anti-proliferative effect of Erlotinib (OSI-774) on human colon cancer cell lines. Cancer Lett. 2005;225:61-74

[32]. Diaz A, Batista AE, and Montero E. Interferon-alpha conditioned sensitivity to an anti-epidermal growth factor receptor monoclonal antibody in a human lung cancer cell line with intermediate expression of the receptor. J Interferon Cytokine Res. 2009:29:433-40.

[33]. Tsaur I, Hudak L, Makarevic J, et al. Intensified antineoplastic effect by combining an HDAC-inhibitor, an mTOR-inhibitor and low dosed interferon alpha in prostate cancer cells. J Cell Mol Med. 2015;19:1795-804.

[34]. Zou X and Blank M. Targeting p38 MAP kinase signaling in cancer through post-translational modifications. Cancer Lett. 2017;384:19-26.

[35]. Roskoski R, Jr. ERK1/2 MAP kinases: structure, function, and regulation. Pharmacol Res. 2012;66:105-43. 